\title{
Chest Computed Tomography Findings and Validation of Clinical Criteria of Stroke Associated Pneumonia
}

\author{
Elena Zapata-Arriaza, ${ }^{a}$ Pilar Serrano-Gotarredona, ${ }^{\mathrm{b}}$ Silvia Navarro-Herrero, ${ }^{\mathrm{b}}$ Francisco Moniche, ${ }^{\mathrm{a}}$ \\ Blanca Pardo-Galiana, ${ }^{\mathrm{a}}$ Esther Pallisa, ${ }^{\mathrm{c}}$ Ángela Vega-Salvatierra, ${ }^{\mathrm{a}}$ Fernando Mancha, ${ }^{\mathrm{a}}$ \\ Irene Escudero-Martínez, ${ }^{\mathrm{a}}$ Alejandro Bustamante, ${ }^{\mathrm{d}}$ Joan Montaner Villalonga ${ }^{\mathrm{a}, \mathrm{e}}$ \\ ${ }^{a}$ Stroke Research Program, Institute of Biomedicine of Seville (IBIS)/ University Hospital Virgen del Rocio/CSIC/University of Seville, Seville, Spain \\ ${ }^{b}$ Department of Radiology, University Hospital Virgen del Rocio, Seville, Spain \\ 'Department of Radiology, Vall d'Hebron University Hospital, Barcelona, Spain \\ ${ }^{\mathrm{d} N e u r o v a s c u l a r ~ R e s e a r c h ~ L a b o r a t o r y, ~ V a l l ~ d ' H e b r o n ~ R e s e a r c h ~ I n s t i t u t e ~(V H I R), ~ B a r c e l o n a, ~ S p a i n ~}$ \\ 'Department of Neurology, University Hospital Virgen Macarena, Seville, Spain
}

\section{Dear Sir:}

Multiple terms have been used to describe chest infection in stroke patients. Pneumonia in Stroke Consensus (PISCES) Group has set stroke associated pneumonia (SAP) diagnostic criteria consensus $^{1}$ as a starting point in the identification of this pathology. However, SAP assessment still involves a particular challenge, ${ }^{2}$ especially if chest radiography is taken into account, due to significant interobserver variability in interpretation and its limited use in the early stages. ${ }^{3}$ In fact, the wide SAP incidence range in stroke units $\left(3.9 \%\right.$ to $\left.44 \%{ }^{4}\right)$ reflects the insufficient evidence about diagnostic accuracy of clinical symptoms or laboratory tests for SAP. ${ }^{2}$ Thorax high resolution computed tomography (THRCT) is a useful adjunct to conventional radiography and an accurate study to identify underlying findings in stroke patients, which could serve as a guide in risk score or diagnostic criteria validation.

To describe for the first time SAP imaging pattern on THRCT, and to evaluate diagnostic performance of SAP clinical criteria based on THRCT findings, we conducted a monocentric, prospective, observational study (approved by our Institutional Review Committee [ID 0103-M1-14]). The patients eligible for the study were those with ischemic stroke and National Institutes of Health Stroke Scale (NIHSS) $\geq 10$ on admission, stroke symptoms onset to inclusion time was $\leq 24$ hours, and informed consent signed by the patient or a relative at inclusion. Mechanical ventilation pneumonia patients transferred from intensive care unit and stroke patients with antibiotic therapy received within last 24 hours before hospital admission were excluded. Dysphagia was evaluated within the first 24 hours with the Acute Stroke

Table 1. Baseline characteristics of the stroke patients that were include in the study and received thorax high resolution computed tomography $(n=41)$

\begin{tabular}{lc}
\hline Variable & Value \\
\hline Male sex & $21(51.2)$ \\
Age (yr) & $75(67-82)$ \\
NIHSS on admission & $20(15-22)$ \\
Time to respiratory infection (day) & $7(3-13)$ \\
Smoker & $12(29.3)$ \\
Ex-smoker & $8(19.5)$ \\
Atrial fibrillation & $19(46.3)$ \\
COPD & $8(19.5)$ \\
Intravenous fibrinolysis & $26(63.4)$ \\
Thrombectomy & $13(31.7)$ \\
Dysphagia & $31(75.6)$ \\
Urinary tract infection & $5(12.1)$ \\
Phlebitis & $1(2.4)$ \\
Clinical respiratory infection (PISCES probable) & $8(19)$ \\
SAP on THRCT (bronchopneumonia) & $5(12.2)$ \\
Any LRTI on THRCT & $8(19.5)$
\end{tabular}

Values are presented as number (\%) or median (interquartile range). NIHSS, National Institutes of Health Stroke Scale; COPD, chronic obstructive pulmonary disease; PISCES, Pneumonia in Stroke Consensus; SAP, stroke associated pneumonia; THRCT, thorax high resolution computed tomography; LRTI, low respiratory tract infection. 


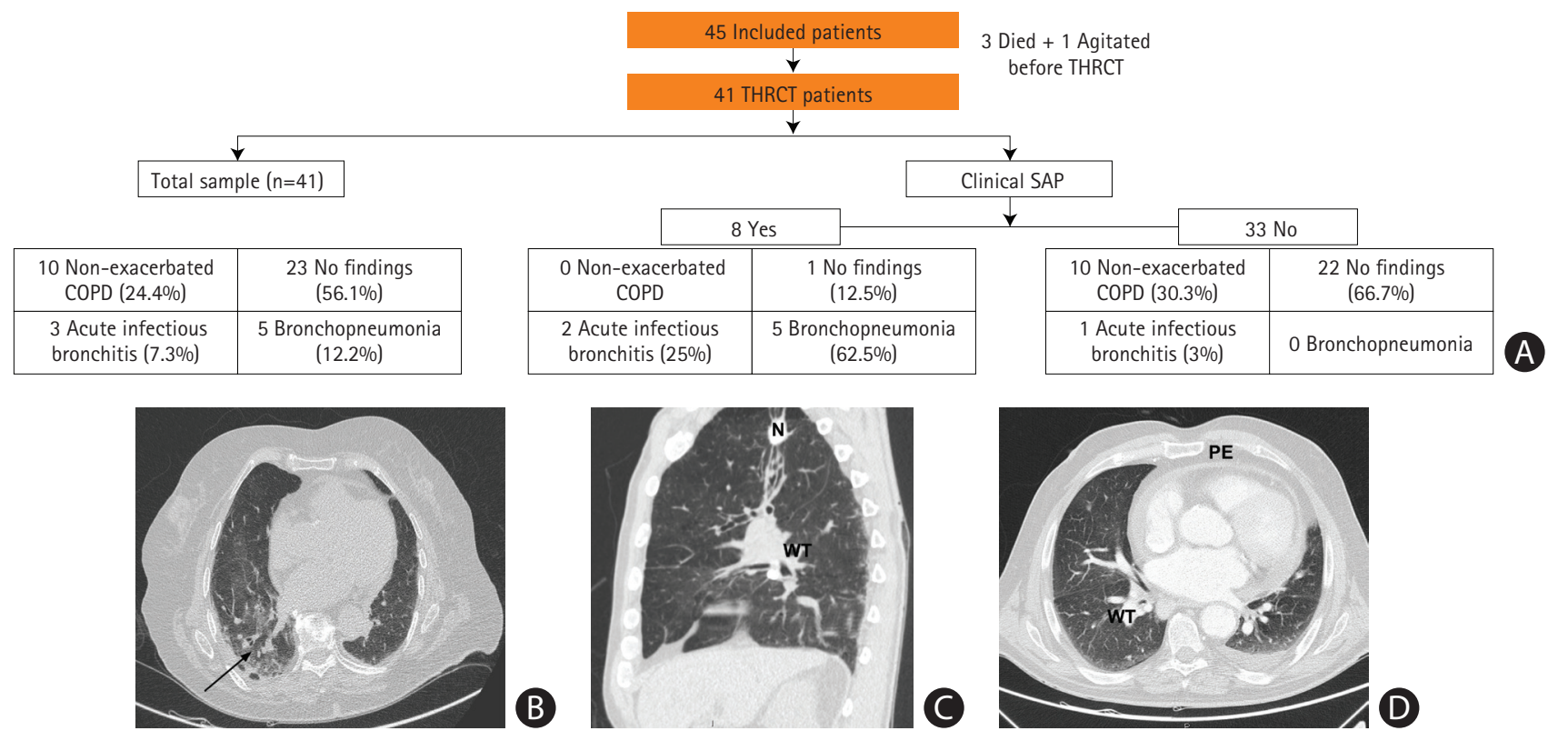

Figure 1. (A) Radiological pattern findings in thorax high resolution computed tomography (THRCT) in the whole sample and regarding clinical stroke associated pneumonia (SAP) ( $n=41)$, including illustration of main radiological findings in the sample. (B) Bronchopneumonia in a patient with clinical suspicion. Right basal consolidation with ground glass opacity adjacent to the consolidating areas of the lower right lobe (black arrow). (C) Infectious bronchitis in a patient with clinical suspicion. Wall thickening with endoluminal secretions. (D) Infectious bronchitis in a patient without clinical suspicion. COPD, chronic obstructive pulmonary disease; PE, pericardial effusion; WT, bronchial wall thickening; N, calcified lung nodule.

Table 2. Sensitivity, specificity, positive predictive value, and negative predictive value of clinical criteria, compared to thorax high resolution computed tomography in any low respiratory tract infection detection and bronchopneumonia

\begin{tabular}{lcccc}
\hline Reference & Sensitivity $(\%)$ & Specificity (\%) & PPV (\%) & NPV (\%) \\
\hline PISCES (probable SAP): any LRTI & 87.5 & 96.9 & 87.5 & 96.9 \\
PISCES (probable SAP): bronchopneumonia & 100 & 91.6 & 62.5 & 100 \\
\hline
\end{tabular}

PPV, positive predictive value; NPV, negative predictive value; PISCES, Pneumonia in Stroke Consensus; SAP, stroke associated pneumonia; LRTI, low respiratory tract infection.

Dysphagia Screen test. ${ }^{5}$ Chest radiography and laboratory test were run following neurologist criteria during admission. A non-contrast THRCT was performed between 5 th and 7th day of admission in all included patients, independently of the suspicion or not of respiratory infection. Every THRCT was evaluated by two skilled thorax radiologists, and discrepancies reviewed by another expert thorax radiologist, all blinded to the clinical data. In this study, SAP was diagnosed by treating physician according to diagnostic categories established by the PISCES criteria. ${ }^{2}$ These categories are probable and definite SAP.

Forty-five patients were included. Three patients died and one presented with psychomotor agitation before THRCT performance, so they were excluded for the study. PISCES probable SAP rate was $19.5 \%(n=8)$. Baseline characteristics of the sample are shown in Table 1. There was no patient with PISCES definite SAP according to treating physician criteria. Moreover, chest radiography low quality was insufficient to a correct in- terpretation of some cases to be considered definitive SAP. The pattern of radiological findings in THRCT of the full sample and regarding probable SAP are shown in Figure 1.

Sensitivity, specificity, positive predictive value, negative predictive values of clinical criteria for any low respiratory tract infection (LRTI) and bronchopneumonia alone are shown in Table 2. Clinical criteria showed high sensitivity and specificity for both any LRTI and bronchopneumonia (87.5\%/96.9\% and 100\%/91.6\%). The $\mathrm{C}$ statistic for clinical criteria was 0.92 (95\% confidence interval $[\mathrm{Cl}], 0.78$ to 1.00$)$ for any LRTI and 0.96 (95\% Cl, 0.89 to 1.00) for bronchopneumonia.

Our study shows for the first time thorax computed tomography (CT) radiological findings of stroke associated respiratory infections, and this allowed us to conduct the first prospective study for external validation of PISCES clinical criteria, using THRCT as an accurate image test to confirm or rule out clinical suspicions. Our SAP rate (12.2\%), identified on thorax CT, remains 
within the limits currently described in a systematic review. ${ }^{6}$

Our cohort is representative of standard stroke care in severe strokes with large vessel occlusions with $63.4 \%$ receiving intravenous thrombolysis and $31.7 \%$ thrombectomy; these reperfusion therapies did not influence SAP rate (Supplementary Table 1).

Clinical presentation of pneumonia in patients who had stroke can be non-specific; since cough is impaired ${ }^{7}$ and sputum microbiological samples have limited availability and poor clinical yield. ${ }^{6}$ The use of chest radiography is recommended by international guidelines ${ }^{1}$ when pneumonia is suspected. However, chest radiography has been demonstrated to be an insensitive method, with $<40 \%$ of new infiltrates on chest radiography in a stroke population ${ }^{8}$ and a low accuracy test. ${ }^{3}$

Some limitations of the present study are small sample size and the low quality of chest radiography, avoiding correct interpretation and making comparison between radiological tests difficult. However, we believe that even with such small sample size our study brings objective data on the descriptive findings of respiratory infections using chest $\mathrm{CT}$ for the first time in stroke patients, showing its usefulness and feasibility.

In fact, this is the first time we can separate bronchitis and lobar condensation, under the same clinical presentation. Probably an increase of sample size will identify even a broader spectrum of findings in SAP. Given high accuracy on thorax CT in SAP diagnosis, a negative THRCT in patients with clinical suspicion, could guide further studies that allow us antibiotic saving, avoiding antibiotic resistance development in hospitalized patients. This study shows that PISCES criteria exhibit a high sensitivity for LRTI and bronchopneumonia detection, defining an interesting tool for screening patients in case of suspected SAP. Although further validation is needed, and multicentric studies will be probably required to get adequate sample size for such type of complex studies, PISCES criteria set an interesting starting point that could be associated with other infection biomarkers to guide future prophylactic antibiotic clinical trials.

\section{Supplementary materials}

Supplementary materials related to this article can be found online at https://doi.org/10.5853/jos.2018.03251.

\section{References}

1. Smith CJ, Kishore AK, Vail A, Chamorro A, Garau J, Hopkins
SJ, et al. Diagnosis of stroke-associated pneumonia: recommendations from the Pneumonia in Stroke Consensus Group. Stroke 2015;46:2335-2340.

2. Kishore AK, Vail A, Chamorro A, Garau J, Hopkins SJ, Di Napoli $M$, et al. How is pneumonia diagnosed in clinical stroke research? A systematic review and meta-analysis. Stroke 2015;46:1202-1209.

3. Hayden GE, Wrenn KW. Chest radiograph vs. computed tomography scan in the evaluation for pneumonia. J Emerg Med 2009;36:266-270.

4. Hannawi Y, Hannawi B, Rao CP, Suarez JI, Bershad EM. Stroke-associated pneumonia: major advances and obstacles. Cerebrovasc Dis 2013;35:430-443.

5. Edmiaston J, Connor LT, Loehr L, Nassief A. Validation of a dysphagia screening tool in acute stroke patients. Am J Crit Care 2010;19:357-364.

6. Kishore AK, Vail A, Jeans AR, Chamorro A, Di Napoli M, Kalra $L$, et al. Microbiological etiologies of pneumonia complicating stroke: a systematic review. Stroke 2018;49:1602-1609.

7. Ward K, Seymour J, Steier J, Jolley CJ, Polkey MI, Kalra L, et al. Acute ischaemic hemispheric stroke is associated with impairment of reflex in addition to voluntary cough. Eur Respir J 2010;36:1383-1390.

8. Kalra L, Hodsoll J, Irshad S, Smithard D, Manawadu D; STROKE-INF Investigators. Comparison of the diagnostic utility of physician-diagnosed with algorithm-defined strokeassociated pneumonia. J Neurol Neurosurg Psychiatry 2016; 87:1163-1168

Correspondence: Joan Montaner Villalonga

Neurovascular Research Laboratory, Institute of Biomedicine of Seville (IBIS),

Calle Antonio Maura Montaner, 41013 Sevilla, Spain

Tel: +34-955923067

Fax: +34-955923101

E-mail:jmontaner-ibis@us.es

Received: November 19, 2018

Revised: January 17, 2019

Accepted: January 18, 2019

This project was partially funded by the ISCIII project PI14/00971. The ITRIBiS project (Improving Translational Research Potential at the Institute of Biomedicine of Seville) has the registration number REGPOT-2013-1. Cooperative Cerebrovascular Disease Research Network (INVICTUS+) (RD16/0019/0015). Fernando Mancha is supported by a Rio Hortega contract. Alejandro Bustamante is supported by a Juan Rodes research contract (JR16/0008) from Instituto de Salud Carlos III.

To the Pneumonia in Stroke Consensus (PISCES) Group and specially to Craig Smith and Andreas Meisel for interesting conversations on the use of chest CT and other biomarkers for the diagnosis of post stroke pneumonia.

The authors have no financial conflicts of interest. 
Supplementary Table 1. Clinical respiratory infection (according to PISCES probable criteria) rate and any low respiratory tract infection on THRCT regarding intravenous fibrinolysis and mechanical thrombectomy

\begin{tabular}{|c|c|c|c|}
\hline Variable & Yes & No & $P$ \\
\hline \multicolumn{4}{|c|}{ Clinical respiratory infection (PISCES probable) } \\
\hline Intravenous fibrinolysis & $5(19.2)$ & $21(80.7)$ & 0.95 \\
\hline Mechanical thrombectomy & $2(15.3)$ & $11(84.6)$ & 0.65 \\
\hline \multicolumn{4}{|c|}{ Any low respiratory tract infection on THRCT } \\
\hline Intravenous fibrinolysis & $6(23.1)$ & $20(76.9)$ & 0.44 \\
\hline Mechanical thrombectomy & $2(15.3)$ & $11(84.6)$ & 0.65 \\
\hline
\end{tabular}

Values are presented as number (\%).

PISCES, Pneumonia in Stroke Consensus; THRCT, thorax high resolution computed tomography. 